\title{
Intensity-Modulated Radiotherapy versus Three-Dimensional Conformal Radiotherapy in Definitive Chemoradiotherapy for Cervical Esophageal Squamous Cell Carcinoma: Comparison of Survival Outcomes and Toxicities
}

\author{
Nai-Bin Chen, MD ${ }^{1,23}$ \\ Bo Oiu, $\mathrm{PhD}^{1,2,3}$ \\ Jun Zhang, MD1,23 \\ Meng-Yun Qiang, MD',2,3 \\ Yu-Jia Zhu, PhD',2,3 \\ Bin Wang, MD1,23 \\ Jin-Yu Guo, BS $1,2,3$ \\ Ling-Zhi Cai, BS1,2,3 \\ Shao-Min Huang, MD ${ }^{1,23}$ \\ Meng-Zhong Liu, PhD1,23 \\ Oun Li, PhD',2,3 \\ Yong-Hong Hu, $\mathrm{PhD}^{1,23}$ \\ Qi-Wen Li, PhD',2,3 \\ Hui Liu, PhD',2,3
} *A list of author's affiliations appears at the
end of the paper.

\section{Correspondence: Hui Liu, PhD}

Department of Radiation Oncology, Sun Yat-sen

University Cancer Center, State Key Laboratory of

Oncology in South China, Collaborative Innovation

Center for Cancer Medicine, 651 Dongfeng Road East,

Guangzhou, Guangdong 510060, P. R. China

Tel: 86-2087343031

Fax: 86-2087343492

E-mail: liuhuisysucc@sina.com.

Co-correspondence: Qi-Wen Li, PhD

Department of Radiation Oncology, Sun Yat-sen

University Cancer Center, State Key Laboratory of

Oncology in South China, Collaborative Innovation

Center for Cancer Medicine, 651 Dongfeng Road East,

Guangzhou, Guangdong 510060, P. R. China

Tel: 86-13660775270

Fax: 86-2087343492

E-mail: liqw@sysucc.org.cn.

Co-correspondence: Yong-Hong $\mathrm{Hu}, \mathrm{PhD}$

Department of Radiation Oncology, Sun Yat-sen

University Cancer Center, State Key Laboratory of

Oncology in South China, Collaborative Innovation

Center for Cancer Medicine, 651 Dongfeng Road East,

Guangzhou, Guangdong 510060, P. R. China

Tel: 86-2087343385

Fax: 86-2087343492

E-mail: huyh@sysucc.org.cn.

Received November 14, 2018

Accepted April 29, 2019

Published Online April 30, 2019

*Nai-Bin Chen and Bo Qiu contributed equally to this work.

\section{Purpose}

The purpose of this study was to compare the survival and toxicities in cervical esophageal squamous cell carcinoma (CESCC) treated by concurrent chemoradiothrapy with either three-dimensional conformal radiotherapy (3D-CRT) or intensity-modulated radiotherapy (IMRT) techniques.

\section{Materials and Methods}

A total of 112 consecutive CESCC patients were retrospectively reviewed. 3D-CRT and IMRT groups had been analyzed by propensity score matching method, with sex, age, Karnofsky performance status, induction chemotherapy, and tumor stage well matched. The KaplanMeier method and Cox proportional hazards model were used for overall survival (OS) and progression-free survival (PFS). Toxicities were compared between two groups by Fisher exact test.

\section{Results}

With a median follow-up time of 34.9 months, the 3-year OS $(p=0.927)$ and PFS $(p=0.859)$ rate was $49.6 \%$ and $45.8 \%$ in 3D-CRT group, compared with $54.4 \%$ and $42.8 \%$ in IMRT group. The rates of grade $\geq 3$ esophagitis, grade $\geq 2$ pneumonitis, esophageal stricture, and hemorrhage were comparable between two groups, while the rate of tracheostomy dependence was much higher in IMRT group than 3D-CRT group (14.3\% vs.1.8\%, p=0.032). Radiotherapy technique (hazard ratio [HR], 0.09; 95\% confidence interval [Cl], 0.01 to 0.79 ) and pretreatment hoarseness ( $\mathrm{HR}, 0.12 ; 95 \% \mathrm{Cl} 0.02$ to 0.70 ) were independently prognostic of tracheostomy dependence.

\section{Conclusion}

No survival benefits had been observed while comparing IMRT versus 3D-CRT in CESCC patients. IMRT with fraction dose escalation and pretreatment hoarseness were considered to be associated with a higher risk for tracheostomy dependence. Radiation dose escalation beyond 60 Gy should be taken into account carefully when using IMRT with hypofractionated regimen. 


\section{Introduction}

Cervical esophageal squamous carcinoma (CESCC) is a relatively rare malignancy, accounting for less than $5 \%$ of all esophageal cancers [1]. Historically, surgical resection has been the mainstay of treatment for CESCC, which requires the removal of the larynx, hypopharynx, and esophagus, and deprives these patients of speech and swallowing. As the notion of organ preservation has been introduced and due to the rapid developments in concurrent chemoradiotherapy (CCRT), physicians and patients predisposed to accept the non-aggressive therapy, hoping to improve functional outcomes after therapy. Prior works [2-13] have documented promising overall survival (OS) and functional preservation with definitive CCRT in CESCC patients.

Three-dimensional conformal radiotherapy (3D-CRT) and intensity-modulated radiotherapy (IMRT) are the most effective and commonly used techniques in esophageal cancer. Although recent studies failed to demonstrate the survival benefit of IMRT based definitive CCRT in esophageal cancer [14,15], IMRT does show a greater advantage in target coverage, dose inhomogeneity, and reducing toxicities to normal organs compared to 3D-CRT [16-19]. With the dosimetric advantage of IMRT technique, hypofractionated radiation therapy for various cancers has been comprehensively studied, which shortens the treatment time and intensifies the dose. Several randomized trials demonstrated that doseescalated, moderately hypofractionated IMRT improves local control in prostate cancer, lung cancer and breast cancer [20-22]. Since previous data suggested that esophageal cancer patients could not benefit from high dose radiation [3], fraction dose escalation (hypofrationated IMRT) started to attract attention especially for CESCC patients.

Since definitive CCRT is an essential treatment option for CESCC, we believed the technique of dose delivery should be well investigated. To our best knowledge, data comparing IMRT with 3D-CRT for CESCC are still limited. Therefore, current study was designed to compare the survival outcomes and toxicities between CESCC patients treated with definitive 3D-CRT and IMRT with concurrent chemotherapy. A propensity score matching (PSM) approach wad used to reduce bias introduced by the non-random treatment assignment, by matching patients with similar clinical stages and other baseline characteristics.

\section{Materials and Methods}

\section{Patient and clinical data}

From January 1, 2000, to September 30, 2016, a total of 138 consecutive patients with CESCC who were treated at the Department of Radiation Oncology, Sun Yat-sen University Cancer Center were retrospectively reviewed. To be included in our study, patients had to meet the following criteria: pathologically confirmed CESSC; treated with definitive radiotherapy with concurrent chemotherapy; without a history of prior radiotherapy; and Karnofsky performance status (KPS) $\geq 70$. Surgical therapy was not allowed to be used. Patients with metastasis upper mediastinal lymph nodes (M1 lymph/stage IV) were included. Each patient underwent a physical examination, laboratory tests, electrocardiogram, lung function test, barium contrast study, endoscopy, and computed tomography (CT) scan of the neck, chest, and upper abdomen. Clinical data collected from each patient included age, sex, KPS, smoking or alcohol-abusing history, primary esophageal tumor location, tumor stage, $\mathrm{T}$ and $\mathrm{N}$ category of primary tumor, total radiation dose, fraction dose, radiotherapy technique, concurrent chemotherapy regimen, pretreatment hoarseness, pretreatment vocal cord paralysis, tracheostomy dependence after treatment, therapeutic toxicities, and tumor progression. Tumor stage was classified using the TNM staging system proposed by the American Joint Committee on Cancer (6th edition).

\section{Treatment}

All patients received external beam radiation, either 3DCRT or IMRT, using a 6-8 MV photon beam, with concurrent chemotherapy. During radiotherapy, immobilization, simulation, and treatment planning were performed according to the standard protocol in our department for patients with cervical esophageal carcinoma [23]. The gross tumor volume (GTV) was defined as visible primary tumors (GTV-T) and involved lymph nodes (GTV-N) on endoscopy, CT and/or positron emission tomography (PET) scans. The criteria of lymph node positivity included: short-axis size $\geq 10 \mathrm{~mm}$, or an infiltrative margin, or central necrosis on pretreatment CT scan, reported positive on the pretreatment PET scan, or biopsy positive. The clinical target volume (CTV) included GTV-T with a 3-cm proximal and distal margin, GTV-N and elective nodal regions, including bilateral levels II-IV of the cervical lymph node area and supraclavicular fossa. The planning target volume (PTV) 1 and PTV2 was defined as a $0.5 \mathrm{~cm}$ margin added to GTV and CTV, respectively. The radiation doses of 60-70 Gy to PTV1 and 46-54 Gy to PTV2 were delivered in 28-35 fractions, with 5 fractions per week. 
Table 1. Demographic and clinical characteristics of the matched patients

\begin{tabular}{|c|c|c|c|}
\hline Characteristic & 3D-CRT $(\mathrm{n}=56)$ & IMRT $(n=56)$ & p-value \\
\hline \multicolumn{4}{|l|}{ Sex } \\
\hline Male & $38(67.9)$ & $38(67.9)$ & $>0.999$ \\
\hline Female & $18(32.1)$ & $18(32.1)$ & \\
\hline \multicolumn{4}{|l|}{ Age (yr) } \\
\hline Median (range) & $60.5(37-81)$ & $58(18-75)$ & 0.706 \\
\hline \multicolumn{4}{|l|}{ KPS } \\
\hline $90-100$ & $36(64.3)$ & $35(62.5)$ & $>0.999$ \\
\hline $70-80$ & $20(35.7)$ & $21(37.5)$ & \\
\hline \multicolumn{4}{|l|}{ Weight loss (\%) } \\
\hline$\geq 10$ & $6(10.7)$ & $4(7.1)$ & 0.521 \\
\hline$<10$ & $43(76.8)$ & $47(83.9)$ & \\
\hline \multicolumn{4}{|c|}{ Pretreatment hoarseness } \\
\hline Yes & $6(10.7)$ & $5(8.9)$ & $>0.999$ \\
\hline No & $50(89.3)$ & $51(91.1)$ & \\
\hline \multicolumn{4}{|c|}{ Pretreatment vocal cord paralysis } \\
\hline Yes & $4(7.1)$ & $3(5.4)$ & $>0.999$ \\
\hline No & $52(92.9)$ & $53(94.6)$ & \\
\hline \multicolumn{4}{|c|}{ T category of primary tumor } \\
\hline T1-2 & $9(16.1)$ & $12(21.4)$ & 0.472 \\
\hline T3-4 & $47(83.9)$ & $42(75.0)$ & \\
\hline \multicolumn{4}{|l|}{ Tumor stage } \\
\hline II & $12(21.4)$ & $12(21.4)$ & 0.131 \\
\hline III & $36(64.3)$ & $42(75.0)$ & \\
\hline IV & $8(14.3)$ & $2(3.6)$ & \\
\hline \multicolumn{4}{|c|}{ Hypopharyngeal extension } \\
\hline Yes & $1(1.8)$ & $16(28.6)$ & $<0.001$ \\
\hline No & $55(98.2)$ & $40(71.4)$ & \\
\hline \multicolumn{4}{|c|}{ Induction chemotherapy } \\
\hline Yes & $11(19.6)$ & $16(28.6)$ & 0.377 \\
\hline No & $45(80.4)$ & $40(71.4)$ & \\
\hline \multicolumn{4}{|c|}{ Concurrent chemotherapy regimen } \\
\hline Single agent & $9(16.1)$ & $10(17.9)$ & $>0.999$ \\
\hline Double agents & $47(83.9)$ & $46(82.1)$ & \\
\hline
\end{tabular}

3D-CRT, three-dimensional conformal radiotherapy; IMRT, intensity-modulated radiotherapy; KPS, Karnofsky performance status.

Concurrent chemotherapy regimens consisted either of a single agent such as 5-fluorouracil or platinum, or of double agents combined with taxane and platinum (TP) or platinum and 5-fluorouracil (PF).

\section{Follow-up and treatment response assessments}

The beginning of the follow-up period was defined as the date of diagnosis. All patients were evaluated weekly during therapy, and then underwent a neck and chest or upper abdomen CT scan every 3 months, and an upper digestive tract endoscopy every 6 months for the first 2 years after completion of CCRT, and subsequently every 6 months thereafter until tumor progression. Bone scans were administered when patients were suspected to have bone metastasis. PET scans were performed in patients with suspected systemic progression. The date of last follow-up was January 1, 2018. Treatment response, OS, and progression-free survival (PFS) were recorded. The responses to CCRT were assessed 2 months after radiotherapy by an independent radiation oncologist according to Response Evaluation Criteria in Solid Tumors ver. 1.1. OS was calculated from the diagnosis of CESCC until death or last follow-up. PFS was defined as the time from diagnosis to tumor recurrence, or 
Table 2. Dosimetric parameters

\begin{tabular}{lccr} 
& \multicolumn{1}{c}{ IMRT } & p-value \\
\hline Total radiation dose (Gy) & 3D-CRT & $63.9(60-70)$ & 0.037 \\
\hline Fraction dose (cGy) & $60(60-70)$ & $213(180-229)$ & $<0.001$ \\
Dmean of GTV (cGy) & $200(165-232)$ & $6,290(5,921-6,455)$ & 0.001 \\
V5 of the lungs (\%) & $6,123(5,939-6,321)$ & $50.2(11.8-88.5)$ & 0.032 \\
V20 of the lungs (\%) & $38.0(18.4-61.9)$ & $21.8(5.5-32.8)$ & 0.479 \\
\hline Dmean of the lungs (cGy) & $18.7(6.1-31.6)$ & $1,101(298-1,658)$ & 0.156 \\
Dmax of the spinal cord (cGy) & $958(488-1,617)$ & $4,344(1,052-4,752)$ & $<0.001$ \\
\hline D5 of PTV1 (cGy) & $4,917(4,030-5,148)$ & $6,745(6,351-7,711)$ & 0.007 \\
\hline Dmean of larynx (cGy) & $6,394(6,150-7,033)$ & $6,045(780-6,902)$ & 0.227 \\
\hline GTV volume $\left(\mathrm{cm}^{3}\right)$ & $5,779(707-6,658)$ & $42.3(4.4-252.0)$ & $>0.999$ \\
\hline
\end{tabular}

Values are presented as median (range). 3D-CRT, three-dimensional conformal radiotherapy; IMRT, intensity-modulated radiotherapy; GTV, gross tumor volume; PTV, planning target volume.
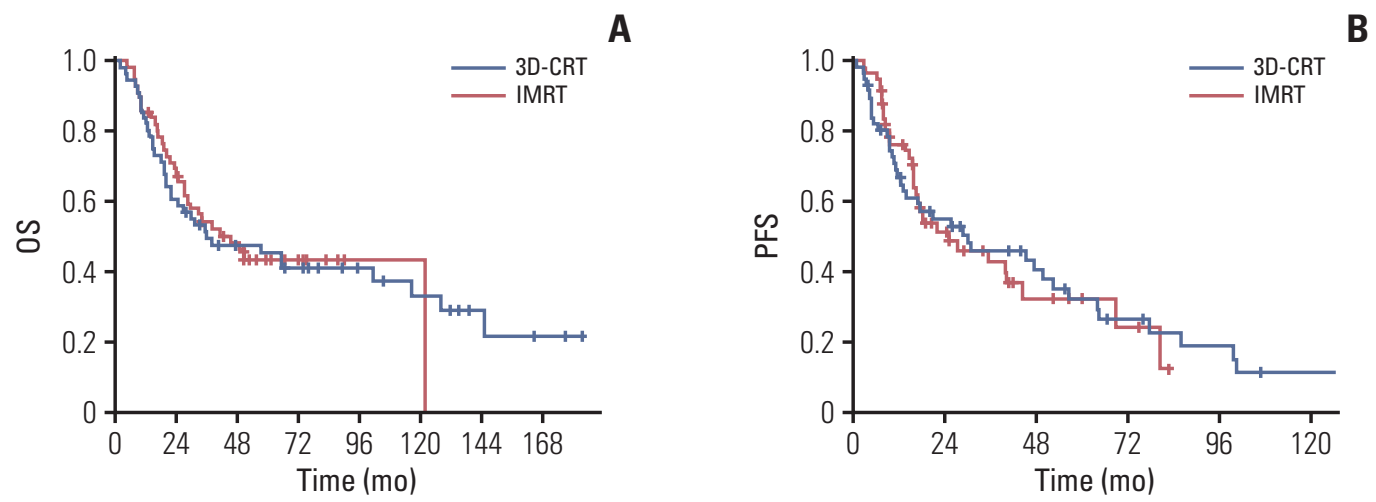

Fig. 1. Overall survival (OS) (A) and progression-free survival (PFS) (B) between the three-dimensional conformal radiotherapy (3D-CRT) and intensity-modulated radiotherapy (IMRT) groups. There was no statistically significant difference observed in OS ( $\mathrm{p}=0.927$, log-rank) and PFS ( $\mathrm{p}=0.859$, log-rank) between the 3D-CRT group and the IMRT group.

death, or last follow-up. OS and PFS were used as measures of survival outcomes. Acute and late toxicities were collected retrospectively and then presented according to The National Cancer Institute Common Toxicity Criteria (ver. 4.0).

\section{Statistical analysis}

We matched patients in the 3D-CRT and IMRT groups by propensity score with a caliper of 0.1 in a 1:1 ratio, using the PSM method with five covariates including sex, age, KPS, induction chemotherapy, and tumor stage. Means, frequencies, and percentage were calculated to describe the data. Continuous variables, such as age, total radiation dose, fraction dose, D5 of PTV1, GTV volume, Dmean of larynx and so on, were normalized as the sample median and then analyzed as nominal categorical variables. Comparisons of categorical variables between groups were first performed by
Fisher exact test, and then the variables that reached a $p$-value $<0.1$ were further evaluated in a multivariate analysis using Logistic regression model. The Kaplan-Meier method was used to produce survival curves. The 3-year, 5-year OS and PFS were estimated. OS and PFS were first compared in univariate analysis by using the two-sided logrank test. Then the Cox proportional hazards model was used to test independent prognostic factors of OS and PFS. All statistical analyses were performed using SPSS ver. 24.0 software (IBM Corp., Armonk, NY), and differences were considered significant at a p-value $<0.05$.

\section{Ethical statement}

Our study was approved by the Ethics Committee of Sun Yat-sen University Cancer Center, and the approval number was YB2017-080. As this was a retrospective analysis of rou- 
Table 3. Univariate analysis of prognostic factors for OS and PFS

\begin{tabular}{|c|c|c|c|c|}
\hline Factor & 3-Year OS (\%) & p-value & 3-Year PFS (\%) & p-value \\
\hline \multicolumn{5}{|l|}{ Sex } \\
\hline Male & 46.4 & 0.088 & 41.3 & 0.058 \\
\hline Female & 63.9 & & 51.2 & \\
\hline \multicolumn{5}{|l|}{ Age (yr) } \\
\hline$<59$ & 55.8 & 0.550 & 49.9 & 0.343 \\
\hline$\geq 59$ & 48.2 & & 38.9 & \\
\hline \multicolumn{5}{|l|}{ KPS } \\
\hline $90-100$ & 54.4 & 0.661 & 45.8 & 0.890 \\
\hline $70-80$ & 52.8 & & 41.6 & \\
\hline \multicolumn{5}{|l|}{ Weight loss (\%) } \\
\hline$\geq 10$ & 36.0 & 0.636 & 30.5 & 0.922 \\
\hline$<10$ & 55.4 & & 44.9 & \\
\hline \multicolumn{5}{|c|}{ Pretreatment hoarseness } \\
\hline Yes & 45.5 & 0.584 & 40.9 & 0.849 \\
\hline No & 52.7 & & 44.8 & \\
\hline \multicolumn{5}{|c|}{ Pretreatment vocal cord paralysis } \\
\hline Yes & 42.9 & 0.650 & 51.4 & 0.508 \\
\hline No & 52.6 & & 44.0 & \\
\hline \multicolumn{5}{|c|}{$\mathrm{T}$ category of primary tumor } \\
\hline $\mathrm{T} 1-2$ & 61.9 & 0.622 & 51.8 & 0.846 \\
\hline T3-4 & 50.1 & & 43.2 & \\
\hline \multicolumn{5}{|l|}{ Tumor stage } \\
\hline II & 61.3 & 0.053 & 49.8 & $<0.001$ \\
\hline III & 53.3 & & 47.0 & \\
\hline IV & 20.0 & & 10.0 & \\
\hline \multicolumn{5}{|c|}{ Hypopharyngeal extension } \\
\hline Yes & 63.0 & 0.289 & 35.9 & 0.894 \\
\hline No & 50.1 & & 45.8 & \\
\hline \multicolumn{5}{|c|}{ Concurrent chemotherapy regimen } \\
\hline Single agent & 47.4 & 0.462 & 26.6 & 0.034 \\
\hline Double agents & 52.9 & & 48.0 & \\
\hline \multicolumn{5}{|c|}{ Induction chemotherapy } \\
\hline Yes & 40.7 & 0.113 & 26.5 & 0.054 \\
\hline No & 55.7 & & 49.8 & \\
\hline \multicolumn{5}{|c|}{ Radiotherapy technique } \\
\hline 3D-CRT & 49.6 & 0.927 & 45.8 & 0.859 \\
\hline IMRT & 54.4 & & 42.8 & \\
\hline \multicolumn{5}{|c|}{ Total radiation dose (Gy) } \\
\hline$<62$ & 55.2 & 0.928 & 43.2 & 0.580 \\
\hline$\geq 62$ & 49.0 & & 45.8 & \\
\hline \multicolumn{5}{|c|}{ Fraction dose (cGy) } \\
\hline$\leq 206$ & 52.6 & 0.664 & 44.0 & 0.701 \\
\hline$>206$ & 51.3 & & 45.3 & \\
\hline \multicolumn{5}{|l|}{ GTV volume $\left(\mathrm{cm}^{3}\right)$} \\
\hline$\leq 43.2$ & 50.9 & 0.988 & 48.0 & 0.453 \\
\hline$>43.2$ & 47.1 & & 30.3 & \\
\hline
\end{tabular}

OS, overall survival; PFS, progression-free survival; KPS, Karnofsky performance status; 3D-CRT, three-dimensional conformal radiotherapy; IMRT, intensity-modulated radiotherapy; GTV, gross tumor volume. 
Table 4. Multivariate analysis of prognostic factors for OS and PFS

\begin{tabular}{lccc} 
Survival outcome & HR & $95 \%$ CI for HR & p-value \\
OS & & & \\
$\quad$ Sex & 0.65 & $0.38-1.11$ & 0.114 \\
$\quad$ Tumor stage & 1.52 & $0.93-2.51$ & 0.098 \\
PFS & & & \\
$\quad$ Sex & 0.60 & $0.36-0.99$ & 0.049 \\
$\quad$ Tumor stage & 1.52 & $0.87-2.65$ & 0.140 \\
$\quad$ Concurrent chemotherapy regimen & 0.59 & $0.31-1.12$ & 0.106 \\
$\quad$ Induction chemotherapy & 1.40 & $0.82-2.37$ & 0.216 \\
\hline
\end{tabular}

OS, overall survival; PFS, progression-free survival; $\mathrm{HR}$, hazard ratio; $\mathrm{CI}$, confidence interval.

Table 5. Univariate analysis of therapeutic toxicities between 3D-CRT and IMRT

\begin{tabular}{lcccc} 
Toxicity & $\begin{array}{c}\text { The whole } \\
\text { group }(\mathbf{n = 1 1 2})\end{array}$ & $\begin{array}{c}\text { 3D-CRT } \\
(\mathbf{n}=56)\end{array}$ & $\begin{array}{c}\text { IMRT } \\
(\mathbf{n}=56)\end{array}$ & p-value \\
Grade $\geq 3$ esophagitis & $60(53.6)$ & $32(57.1)$ & $28(50.0)$ & 0.570 \\
Grade $\geq 2$ pneumonitis & $8(7.1)$ & $3(5.4)$ & $5(8.9)$ & 0.716 \\
Esophageal stricture & $19(17.0)$ & $12(21.4)$ & $7(12.5)$ & 0.314 \\
Life-threatening hemorrhage & $2(1.8)$ & $1(1.8)$ & $1(1.8)$ & $>0.999$ \\
Tracheostomy & $9(8.0)$ & $1(1.8)$ & $8(14.3)$ & 0.032 \\
\hline
\end{tabular}

3D-CRT, three-dimensional conformal radiotherapy; IMRT, intensity-modulated radiotherapy.

tine clinical data, a waiver of the requirement for individual informed consent was granted by our institutional ethics committee.

\section{Results}

\section{Baseline characteristics}

A total of 112 of the included 138 consecutive patients were divided into the 3D-CRT and IMRT groups based on the above-mentioned PSM procedure. Patient sex, age, KPS, induction chemotherapy, and tumor stage were well matched ( $p>0.1)$. The demographic and clinical characteristics of the cohort are listed in Table 1 . The median age was 59 years, range from 18 to 81 . Most patients $(102 / 112,77 \%)$ had stages II and III disease and the rest 10 patients had stages IV disease. As for T category, 21 patients had T1-2 lesion, and 89 had T3-4 lesion. In our group, 9.8\% (11/112) patients had pretreatment hoarseness, and the incidence of pretreatment vocal cord paralysis was $6.3 \%$ (7/112). The median radiation dose was 62 Gy (range, 60 to 70 Gy) in 30-35 fractions. The dosimetric parameters were listed in Table 2. The median fraction dose, Dmean of GTV, V5 of the lungs, V20 of the lungs, Dmean of the lungs, Dmax of the spinal cord, D5 of PTV1 and Dmean of larynx in the 3D-CRT group vs. IMRT group was 200 cGy vs. 213 cGy (p < 0.001, Fisher exact test), 6,123 cGy vs. 6,290 cGy ( $\mathrm{p}=0.001$ ), 38.0 vs. 50.2 ( $\mathrm{p}=0.032), 18.7$ vs. 21.8 ( $\mathrm{p}=0.479), 958$ cGy vs. $1,101 \mathrm{cGy}(\mathrm{p}=0.156), 4,917 \mathrm{cGy}$ vs. 4,344 cGy ( $\mathrm{p}<0.001), 6,394$ cGy vs. 6,745 cGy ( $\mathrm{p}=0.007)$, and 5,779 cGy vs. 6,045 cGy ( $\mathrm{p}=0.227)$, respectively. Most patients $(93 / 112,83 \%)$ received double agents chemotherapy regimens, and the remaining adopted single-agent regimens. Part of patients $(27 / 112,24 \%)$ underwent induction chemotherapy before definitive CCRT.

\section{Survival outcomes}

With a median follow-up of 34.9 months (range, 2.1 to 183.6 months), our analysis demonstrated a median estimated OS of 36.0 months in the 3D-CRT group, 45.6 months in the IMRT group and 41.4 months in the whole group. The 3 -year and 5-year OS rate was $49.6 \%$ and $45.6 \%$ in the 3DCRT group, compared with $54.4 \%$ and $43.8 \%$ in the IMRT group ( $\mathrm{p}=0.927$, log-rank). The median estimated PFS was 30.2, 25.2, and 27.5 months in the 3D-CRT, IMRT, and the 
Table 6. Univariate analysis of prognostic factors for tracheostomy dependence

\begin{tabular}{|c|c|c|}
\hline Factor & Incidence $(\%)$ & p-value \\
\hline \multicolumn{3}{|l|}{ Sex } \\
\hline Male & 9.2 & 0.716 \\
\hline Female & 5.6 & \\
\hline \multicolumn{3}{|l|}{ Age (yr) } \\
\hline$\leq 59$ & 9.1 & 0.740 \\
\hline$>59$ & 7.0 & \\
\hline \multicolumn{3}{|l|}{ KPS } \\
\hline $90-100$ & 7.0 & 0.722 \\
\hline $70-80$ & 9.8 & \\
\hline \multicolumn{3}{|l|}{ Weight loss (\%) } \\
\hline$\geq 10$ & 0.0 & $>0.999$ \\
\hline$<10$ & 7.8 & \\
\hline \multicolumn{3}{|c|}{ Pretreatment hoarseness } \\
\hline Yes & 27.3 & 0.043 \\
\hline No & 5.9 & \\
\hline \multicolumn{3}{|c|}{ Pretreatment vocal cord paralysis } \\
\hline Yes & 28.5 & 0.098 \\
\hline No & 6.7 & \\
\hline \multicolumn{3}{|c|}{ T category of primary tumor } \\
\hline T1-2 & 0.0 & 0.349 \\
\hline T3-4 & 9.0 & \\
\hline \multicolumn{3}{|l|}{ Tumor stage } \\
\hline II & 8.3 & 0.616 \\
\hline III & 9.0 & \\
\hline IV & 0.0 & \\
\hline \multicolumn{3}{|c|}{ Hypopharyngeal extension } \\
\hline Yes & 11.8 & 0.624 \\
\hline No & 7.4 & \\
\hline \multicolumn{3}{|c|}{ Concurrent chemotherapy regimen } \\
\hline Single agent & 5.3 & $>0.999$ \\
\hline Double agents & 8.6 & \\
\hline \multicolumn{3}{|c|}{ Induction chemotherapy } \\
\hline Yes & 3.7 & 0.685 \\
\hline No & 9.4 & \\
\hline \multicolumn{3}{|c|}{ Radiotherapy technique } \\
\hline 3D-CRT & 1.8 & 0.032 \\
\hline IMRT & 14.3 & \\
\hline \multicolumn{3}{|c|}{ Total radiation dose (Gy) } \\
\hline$<62$ & 3.7 & 0.164 \\
\hline$\geq 62$ & 12.1 & \\
\hline \multicolumn{3}{|c|}{ Fraction dose (cGy) } \\
\hline$<206$ & 3.5 & 0.091 \\
\hline$\geq 206$ & 12.7 & \\
\hline \multicolumn{3}{|l|}{ PTV1 D5 (cGy) } \\
\hline$\leq 6,542$ & 5.7 & 0.428 \\
\hline$>6,542$ & 11.8 & \\
\hline
\end{tabular}

(Continued)
Table 6. Continued

\begin{tabular}{lcc} 
Factor & Incidence $(\%)$ & p-value \\
GTV volume $\left(\mathrm{cm}^{3}\right)$ & & \\
$\quad \leq 43.2$ & 11.4 & 0.673 \\
$\quad>43.2$ & 5.9 & \\
$\quad$ Dmean of larynx (cGy) & & \\
$\quad \leq 5,908$ & 8.6 & $>0.999$ \\
$>5,908$ & 8.8 & \\
\hline
\end{tabular}

KPS, Karnofsky performance status; 3D-CRT, threedimensional conformal radiotherapy; IMRT, intensitymodulated radiotherapy; PTV, planning target volume; GTV, gross tumor volume.

whole group, respectively. And the 3-year and 5-year PFS rate was $45.8 \%$ and $32.1 \%$ in the 3D-CRT group, vs. $42.8 \%$ and $32.1 \%$ in the IMRT group ( $\mathrm{p}=0.859$, log-rank) (Fig. 1). There was no statistically significant difference observed in OS and PFS between the 3D-CRT group and the IMRT group.

\section{Prognostic factors}

Univariate Kaplan-Meier survival analysis found that sex $(\mathrm{p}=0.088)$ and tumor stage $(\mathrm{p}=0.053)$ were significantly associated with OS (Table 3). Multivariate Cox proportional hazards model identified none of them reached statistical significance (Table 4). Similarly, univariate analysis of factors influencing PFS showed that sex $(\mathrm{p}=0.058)$, concurrent chemotherapy regimen $(\mathrm{p}=0.034)$, induction chemotherapy $(p=0.054)$ and tumor stage $(p<0.001)$ were statistically significant variables. Only sex remained independently related to PFS in multivariable analysis (hazard ratio [HR], 0.60; 95\% confidence interval [CI], 0.36 to 0.99 ).

\section{Toxicities}

The most commonly documented therapeutic toxicity was radiation esophagitis, and most patients $(60 / 112,53.6 \%)$ had grade 3 esophagitis. Seventeen percent of patients (19/112) had esophageal stricture and required dilatation, with 12 in the 3-dimensional radiotherapy (3D-RT) group and seven in the IMRT group. Other observed toxicities were mostly grade 1 or 2, including radiation pneumonitis and gastrointestinal toxicity. There were both one reported case of lifethreatening hemorrhage in the 3D-CRT group and the IMRT group. However, none of these toxicities reached statistical significance between the 3D-RT group and the IMRT group (Table 5). No patient developed acute grade 4 toxicity, and there were no treatment-related deaths. 
Table 7. Multivariate analysis of prognostic factors for tracheostomy dependence

\begin{tabular}{lccc} 
Factor & HR & $95 \%$ CI for HR & p-value \\
Radiotherapy technique & 0.09 & $0.01-0.79$ & 0.030 \\
Pretreatment hoarseness & 0.12 & $0.02-0.70$ & 0.018 \\
\hline
\end{tabular}

$\mathrm{HR}$, hazard ratio; $\mathrm{CI}$, confidence interval.

Moreover, we observed that $8.0 \%$ of patients $(9 / 112)$ developed dyspnea and required tracheostomy within 6 months after cessation of therapy, without any evidence of tumor progression or second primary tumor. Univariate analysis indicated that radiotherapy $(\mathrm{RT})$ technique $(\mathrm{p}=0.032)$, fraction dose $(\mathrm{p}=0.091)$, pretreatment hoarseness $(\mathrm{p}=0.043)$, and pretreatment vocal cord paralysis $(\mathrm{p}=0.098)$ were significantly related to tracheostomy dependence (Table 6). IMRT, fraction dose more than $206 \mathrm{cGy}$, pretreatment hoarseness, and pretreatment vocal cord paralysis were risk factors for tracheostomy dependence. Since fraction dose was significantly associated with RT technique $(\mathrm{p}<0.001)$, and pretreatment vocal cord paralysis was significantly associated with pretreatment hoarseness $(\mathrm{p}<0.001)$, we excluded fraction dose and pretreatment vocal cord paralysis from multivariate analysis, then the other two variables reached $p<0.1$ in univariate analysis were further analyzed by using a Logistic regression model. RT technique (HR, $0.09 ; 95 \% \mathrm{CI}, 0.01$ to 0.79 ) and pretreatment hoarseness (HR, $0.12 ; 95 \% \mathrm{CI}, 0.02$ to 0.70 ) were independently prognostic of tracheostomy dependence (Table 7).

\section{Discussion}

Although dosimetric studies [16-19] reported that IMRT was better than 3D-CRT with respect to improved target coverage and conformality, the clinical benefits of IMRT have not been well established. To our knowledge, this is the first study to compare the survival outcomes and toxicities of CESCC between the 3D-CRT and the IMRT group using PSM approach with the largest sample size. Our study indicated OS and PFS were comparable between the 3D-CRT and IMRT groups. The 3-year and 5-year OS rate was $49.6 \%$ and $45.6 \%$ in the 3D-CRT group, while $54.4 \%$ and $43.8 \%$ in the IMRT group ( $\mathrm{p}=0.927, \mathrm{log}$-rank). The 3-year and 5-year PFS rate was $45.8 \%$ and $32.1 \%$ in the 3D-CRT group, vs. $42.8 \%$ and $32.1 \%$ in the IMRT group ( $\mathrm{p}=0.859, \log$-rank).

Advances in radiotherapy technique in treatment planning and radiation delivery, such as simultaneous integrated boosting IMRT, could allow the optimization of target vol- ume coverage, which theoretically resulted in better survival outcomes. However, the published data failed to support the significant advantage of IMRT in survival. Yang et al. [24] reported that OS and failure-free survival (FFS) were not significantly different between the IMRT and 3D-CRT group, with the 2-year OS and FFS for the 3D-CRT vs. IMRT group was $53.6 \%$ vs. $55.6 \%$ ( $\mathrm{p}=0.965)$, and $49.5 \%$ vs. $56.7 \%$ ( $\mathrm{p}=0.998$ ), respectively. Similarly, there was no statistically significant difference observed in OS, local FFS, and regional FFS between the IMRT group and two-dimensional conformal radiotherapy group in the study conducted by Cao et al. [25]. A recent published data from Japan [26] showed that IMRT achieved a significantly better 3-year OS than 3D-CRT (81.6\% vs. $57.2 \%, \mathrm{p}=0.037)$. They found that the sufficient salvage rate was higher in IMRT than 3D-CRT group when locoregional recurrence was presented, which contributed to the difference in OS. There was yet no statistical difference in locoregional control or PFS between these two groups. Similarly to most of the previous studies, our study did not support the survival advantage of IMRT compared with 3D-CRT. In our study, the Dmean of GTV was higher in IMRT group $(6,290 \mathrm{cGy}$ vs. 6,123 cGy, $\mathrm{p}=0.001)$. It suggests that there was no more dose-response relationship in dose ranges over 50 Gy, as indicated by series of Radiation Therapy Oncology Group studies [2,3].

IMRT could achieve well-demonstrated sparing of organs at risk, such as lungs and spinal cord, reducing both acute and late toxicities. However, our analysis showed that the rates of observed toxicities, including pneumonitis, esophagitis, esophageal stricture, gastrointestinal reaction, and lifethreatening hemorrhage were comparable between the 3DCRT group and the IMRT group. Detailed dosimetric study showed similar V20 and mean dose of the lungs between two groups, a relatively lower Dmax of spinal cord and a higher V5 of the lungs in the IMRT group. These results indicated that both 3DCRT and IMRT could be delivered safely without obvious toxicities when the total dose did not exceed 70 Gy.

Within 6 months after CCRT, nine patients $(8 \%, 9 / 112)$ developed dyspnea and required tracheostomy. All of the nine patients got partial remission after therapy and did not show any evidence of tumor progression at the time of tracheostomy. Few published reports had recorded and ana- 
lyzed this complication. Gkika et al. [13] reported one patient $(1.8 \%, 1 / 55)$ with CESCC underwent tracheostomy during definitive CCRT because of inability to breathe, who had got a paralysis of the recurrent laryngeal nerve before treatment. In Tong's study [4], two patients $(9.5 \%, 2 / 21)$ treated with up-front CCRT had bilateral vocal cord palsy requiring permanent tracheostomy. Staton et al. [27] reviewed 45 patients with advanced laryngeal cancer at 6 months after CCRT, and they found that the rate of tracheostomy dependence was $44 \%$ in patients with baseline vocal fold fixation versus $6 \%$ in patients without fixation. The authors attributed tracheostomy dependence to radiation-related bilateral recurrent laryngeal nerve paralysis, which results in bilateral vocal cord fixation. Similarly, in our cohort, pretreatment hoarseness was an independent risk factor for tracheostomy dependence during or after CCRT. As a result, it is urgent to call for close surveillance in those with pretreatment hoarseness or vocal cord paralysis during and 6 months after therapy. Our results showed a significantly higher rate of tracheostomy in IMRT group than that in 3D-CRT group (14.3\% vs. $1.8 \%, \mathrm{p}=0.032$ ). Most IMRT plans in our cohort utilize the SIB technique with the median fraction dose to GTV of 213 cGy. For patients with CESSC, recurrent laryngeal nerves are always located in the proximity of GTV, which inevitably received a fraction dose more than 200 cGy when treated with IMRT. Therefore, we inferred that IMRT with fraction dose escalation (hypofractionated IMRT) might lead to a higher rate for tracheostomy dependence. Hypofractionated IMRT scheme should be carefully defined especially with concurrent chemotherapy in case of severe adverse events.

This retrospective study has several limitations, such as selection bias and relatively small number of patients which might affect the results of our study, and it was a singleinstitution experience. Larger multi-center prospective trials are warranted to validate our results.

No survival benefits had been observed while comparing IMRT versus 3D-CRT in CESCC patients. Sex was the only independent prognostic factor for PFS. IMRT with fraction dose escalation (hypofractionated IMRT) was considered to be associated with a higher risk for tracheostomy dependence within 6 months after definitive CCRT, and patients with pretreatment hoarseness or vocal cord paralysis should be under close surveillance for dyspnea. Radiation dose escalation beyond $60 \mathrm{~Gy}$ should be taken into account carefully when using IMRT with hypofractionated regimen. Prospective studies are warranted to verify presented results.

\section{Conflicts of Interest}

Conflict of interest relevant to this article was not reported.

\section{Acknowledgments}

Our study was supported by Guangdong Esophageal Cancer Institute Science and Technology Program (M201505).

\section{Author Details}

${ }^{1}$ Department of Radiation Oncology, Sun Yat-sen University Cancer Center, Guangzhou, ${ }^{2}$ State Key Laboratory of Oncology in South China, Guangzhou, ${ }^{3}$ Collaborative Innovation Center for Cancer Medicine, Guangzhou, China

\section{References}

1. Mendenhall WM, Sombeck MD, Parsons JT, Kasper ME, Stringer SP, Vogel SB. Management of cervical esophageal carcinoma. Semin Radiat Oncol. 1994;4:179-91.

2. Cooper JS, Guo MD, Herskovic A, Macdonald JS, Martenson JA Jr, Al-Sarraf M, et al. Chemoradiotherapy of locally advanced esophageal cancer: long-term follow-up of a prospective randomized trial (RTOG 85-01). Radiation Therapy Oncology Group. JAMA. 1999;281:1623-7.

3. Minsky BD, Pajak TF, Ginsberg RJ, Pisansky TM, Martenson J, Komaki R, et al. INT 0123 (Radiation Therapy Oncology Group 94-05) phase III trial of combined-modality therapy for esophageal cancer: high-dose versus standard-dose radiation therapy. J Clin Oncol. 2002;20:1167-74.

4. Tong DK, Law S, Kwong DL, Wei WI, Ng RW, Wong KH. Current management of cervical esophageal cancer. World J Surg. 2011;35:600-7.
5. Kim HJ, Suh YG, Lee YC, Lee SK, Shin SK, Cho BC, et al. Doseresponse relationship between radiation dose and locoregional control in patients with stage II-III esophageal cancer treated with definitive chemoradiotherapy. Cancer Res Treat. 2017;49:669-77.

6. Grass GD, Cooper SL, Armeson K, Garrett-Mayer E, Sharma A. Cervical esophageal cancer: a population-based study. Head Neck. 2015;37:808-14.

7. Yamada K, Murakami M, Okamoto Y, Okuno Y, Nakajima T, Kusumi F, et al. Treatment results of radiotherapy for carcinoma of the cervical esophagus. Acta Oncol. 2006;45:1120-5.

8. Wang S, Liao Z, Chen Y, Chang JY, Jeter M, Guerrero T, et al. Esophageal cancer located at the neck and upper thorax treated with concurrent chemoradiation: a single-institution experience. J Thorac Oncol. 2006;1:252-9.

9. Huang SH, Lockwood G, Brierley J, Cummings B, Kim J, 
Wong R, et al. Effect of concurrent high-dose cisplatin chemotherapy and conformal radiotherapy on cervical esophageal cancer survival. Int J Radiat Oncol Biol Phys. 2008;71: 735-40.

10. Zhang P, Xi M, Zhao L, Qiu B, Liu H, Hu YH, et al. Clinical efficacy and failure pattern in patients with cervical esophageal cancer treated with definitive chemoradiotherapy. Radiother Oncol. 2015;116:257-61.

11. Cao C, Luo J, Gao L, Xu G, Yi J, Huang X, et al. Definitive radiotherapy for cervical esophageal cancer. Head Neck. 2015; 37:151-5.

12. Zhao L, Zhou Y, Mu Y, Chai G, Xiao F, Tan L, et al. Patterns of failure and clinical outcomes of definitive radiotherapy for cervical esophageal cancer. Oncotarget. 2017;8:21852-60.

13. Gkika E, Gauler T, Eberhardt W, Stahl M, Stuschke M, Pottgen C. Long-term results of definitive radiochemotherapy in locally advanced cancers of the cervical esophagus. Dis Esophagus. 2014;27:678-84

14. Freilich J, Hoffe SE, Almhanna K, Dinwoodie W, Yue B, Fulp $\mathrm{W}$, et al. Comparative outcomes for three-dimensional conformal versus intensity-modulated radiation therapy for esophageal cancer. Dis Esophagus. 2015;28:352-7.

15. Haefner MF, Lang K, Verma V, Koerber SA, Uhlmann L, Debus J, et al. Intensity-modulated versus 3-dimensional conformal radiotherapy in the definitive treatment of esophageal cancer: comparison of outcomes and acute toxicity. Radiat Oncol. 2017;12:131.

16. Nicolini G, Ghosh-Laskar S, Shrivastava SK, Banerjee S, Chaudhary S, Agarwal JP, et al. Volumetric modulation arc radiotherapy with flattening filter-free beams compared with static gantry IMRT and 3D conformal radiotherapy for advanced esophageal cancer: a feasibility study. Int J Radiat Oncol Biol Phys. 2012;84:553-60.

17. Zhang WZ, Chen JZ, Li DR, Chen ZJ, Guo H, Zhuang TT, et al. Simultaneous modulated accelerated radiation therapy for esophageal cancer: a feasibility study. World J Gastroenterol. 2014;20:13973-80.

18. Fenkell L, Kaminsky I, Breen S, Huang S, Van Prooijen M, Ringash J. Dosimetric comparison of IMRT vs. 3D conformal radiotherapy in the treatment of cancer of the cervical esophagus. Radiother Oncol. 2008;89:287-91.

19. Gao M, Li Q, Ning Z, Gu W, Huang J, Mu J, et al. Dosimetric comparison between step-shoot intensity-modulated radiotherapy and volumetric-modulated arc therapy for upper thoracic and cervical esophageal carcinoma. Med Dosim. 2016;41:131-5.

20. Hoffman KE, Voong KR, Levy LB, Allen PK, Choi S, Schlembach PJ, et al. Randomized trial of hypofractionated, doseescalated, intensity-modulated radiation therapy (IMRT) versus conventionally fractionated IMRT for localized prostate cancer. J Clin Oncol. 2018;36:2943-9.

21. Kim JO, Chu KP, Fairchild A, Ghosh S, Butts C, Chu Q, et al. Dose-escalated hypofractionated intensity-modulated radiation therapy with concurrent chemotherapy for inoperable or unresectable non-small cell lung cancer. Am J Clin Oncol. 2017;40:294-9.

22. Freedman GM, Anderson PR, Bleicher RJ, Litwin S, Li T, Swaby RF, et al. Five-year local control in a phase II study of hypofractionated intensity modulated radiation therapy with an incorporated boost for early stage breast cancer. Int J Radiat Oncol Biol Phys. 2012;84:888-93.

23. Li QQ, Liu MZ, Hu YH, Liu H, He ZY, Lin HX. Definitive concomitant chemoradiotherapy with docetaxel and cisplatin in squamous esophageal carcinoma. Dis Esophagus. 2010;23: 253-9.

24. Yang H, Feng C, Cai BN, Yang J, Liu HX, Ma L. Comparison of three-dimensional conformal radiation therapy, intensitymodulated radiation therapy, and volumetric-modulated arc therapy in the treatment of cervical esophageal carcinoma. Dis Esophagus. 2017;30:1-8.

25. Cao C, Luo J, Gao L, Xu G, Yi J, Huang X, et al. Definitive intensity-modulated radiotherapy compared with definitive conventional radiotherapy in cervical oesophageal squamous cell carcinoma. Radiol Med. 2015;120:603-10.

26. Ito M, Kodaira T, Tachibana H, Tomita N, Makita C, Koide $Y$, et al. Clinical results of definitive chemoradiotherapy for cervical esophageal cancer: comparison of failure pattern and toxicities between intensity-modulated radiotherapy and 3-dimensional conformal radiotherapy. Head Neck. 2017;39: 2406-15.

27. Staton J, Robbins KT, Newman L, Samant S, Sebelik M, Vieira F. Factors predictive of poor functional outcome after chemoradiation for advanced laryngeal cancer. Otolaryngol Head Neck Surg. 2002;127:43-7. 